\title{
High-Order Structure and Flame Retardation Effect of Poly(tetrafluoroethylene) in Thermoplastic Resin Compositions
}

\author{
Hajime NishiHaRa and Susumu TaNJI \\ Department of Styrenic Resins Development, Asahi Chemical Industry Co., Ltd., \\ Kawasaki, Kanagawa, 210-0863, Japan
}

(Received September 17, 1997)

\begin{abstract}
Melt-kneading of a high-viscosity resin, such as polyphenylene ether, together with poly(tetrafluoroethylene) (PTFE) at high shear rate and temperature just above the melting point of PTFE [ $323^{\circ} \mathrm{C}$ (by DSC)] results in the formation of PTFE in a branched fibril form comprising oriented trunk and branch fibers, in diameter $0.15 \mu \mathrm{m}$. When a low-viscosity resin, such as rubber-modified polystyrene (HIPS), is melt-kneaded with PTFE at temperature lower than the PTFE melting point, PTFE in linear fibril form having a diameter of $0.05 \mu \mathrm{m}$ and without branch fibers is formed. Molded resins containing the branched-fibril PTFE undergo larger dimensional changes than those containing the linear-fibril PTFE, when subjected to heat treatment. This indicates higher orientation of the former PTFE, and thus greater enhancement of flame retardancy in resin compositions containing HIPS, polyphenylene ether (PPE), and a phosphorus-based flame retardant.

KEY WORDS Poly(tetrafluoroethylene) / High-Order Structure / Orientation / Flame Retardancy / Styrenic Resin / Polyphenylene Ether / Melting Point / Fibril /
\end{abstract}

Thermoplastic resins have widely replaced conventionally used materials such as wood, glass, and metals in many fields including automobiles, household appliances, and office equipment-but with restrictions due to their flammability in applications where flame retardancy is required.

Increasingly high levels of flame retardation as rated by the Underwriters' Laboratory (UL) have become required for plastic materials used in household appliances and office equipment.

The pursuit of materials economy by manufacturers and preference for lightweight products on the part of end-product users have led to increasing use of thin-walled plastic parts, prone to flame dripping during combustion and thereby contributing to the spread of fire to other materials.

These circumstances have prompted the development of advanced flame retardation technologies, particularly those for flame dripping prevention. The addition of poly(tetrafluoroethylene) (PTFE), for example, is an effective means to prevent flame dripping from plastics, such as styrenic resins and polyphenylene ether (PPE), during their combustion. ${ }^{1-3}$ We further enhanced flame retardation of PTFE for thermoplastic resin compositions, by controlling its morphology.

This paper describes to the relationship between PTFE morphology and flame retardancy of PTFE-containing resins by melt-kneading with various compositions and conditions, and transmission electron microscopy, and evaluating flame-retardance properties.

\section{EXPERIMENTAL}

\section{Materials}

We used commercial non-modified polystyrene as GPPS; a polybutadiene modified polystyrene (polystyrene : polybutadiene $=88: 12$ by weight) as HIPS; and poly(oxy-2,6-dimethyl-1,4-phenylene) as PPE. All three resins are manufactured and sold by Asahi Chemical Industry Co., Ltd., Japan. Melamine cyanurate (MC) manufactured by Nissan Chemical Industry Co., Ltd., Japan, was used as the nitrogen-containing flame retardant. Teflon $6 \mathbf{J}$ manufactured by Mitsui-Fluorochemical Co., Ltd., Japan, was used as PTFE. Ethylene bis(stearamide) (EBS) manufactured by Kao Co., Ltd., Japan, was used as the dispersing agent.

Resorcinyl diphenyl phosphate (TPP-OH) was prepared as follows. ${ }^{4,5}$

A flask was charged with 122.7 parts phenol and 0.87 part aluminum chloride, and 100 parts phosphorus oxychloride were added dropwise at $90^{\circ} \mathrm{C}$ over $1 \mathrm{~h}$ (molar ratio of phenol:aluminum chloride: phosphorus oxychloride $=2.0: 0.01: 1.0$ ). To the intermediate product thus formed was added 71.7 parts (molar ratio, 1.0) resorcinol to continue the reaction. The reaction mixture was gradually heated to $180^{\circ} \mathrm{C}$ to complete esterification. The reaction product was cooled and washed with water. The catalyst and chlorine were removed to obtain a phosphoric ester mixture (designated FR-1). Gel-permeation chromatography (GPC) showed the mixture to contain resorcinyl diphenyl phosphate, triphenyl phosphate, and resorcinol bis(diphenyl phosphate) at $54.2: 18.3: 27.5$. The mixture (FR1) was distilled, and fractionated by liquid chromatography to obtain TPP-OH.

\section{Preparation of Specimens}

The components of each resin shown in Tables I and II were mechanically mixed at the ratios, and the mixture was melt-kneaded in a Laboplastomill batch mixer manufactured by Toyo Seiki Seisakusho. The resulting resin was compression molded to prepare specimens.

\section{Measurements}

- Flame retardancy: Evaluated by a vertical burning (VB) test in accordance with UL-94, with $1 / 8$-inch-thick specimens.

- Melting point of PTFE: Measured by differential scanning calorimetry (DSC) while heating PTFE at a rate of $10^{\circ} \mathrm{C} \mathrm{min}^{-1}$ in a stream of nitrogen by means 
of Type DT-40 thermal analyzer from Shimadzu Corp. of Japan (Figure 1).

- Morphology of PTFE: ${ }^{6}$ Examined by transmission electron microscopy (TEM) as follows. A $0.1 \mathrm{~mm}$ square or smaller piece was cut out from a compression-molded sheet of the PTFE-containing resin, and an ultrathin slice was cut therefrom with a diamond knife. The slice was exposed to the vapor of aqueous $1 \%$ ruthenic acid solution in a sealed container for several hours under light-shielded conditions, and stained with a dye and then subjected to TEM observation to determine the morphology of PTFE.

\section{RESULTS AND DISCUSSION}

PTFE Melt-Kneading Conditions and Resulting Morphology

PTFE is stable at high temperatures, retaining consid-

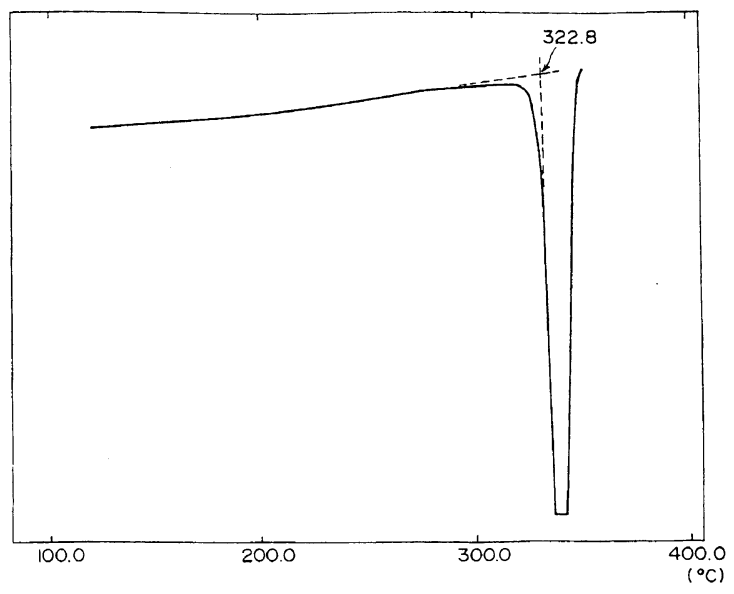

Temperature $\left({ }^{\circ} \mathrm{C}\right)$

Figure 1. Melting point of PTFE by DSC.

a)

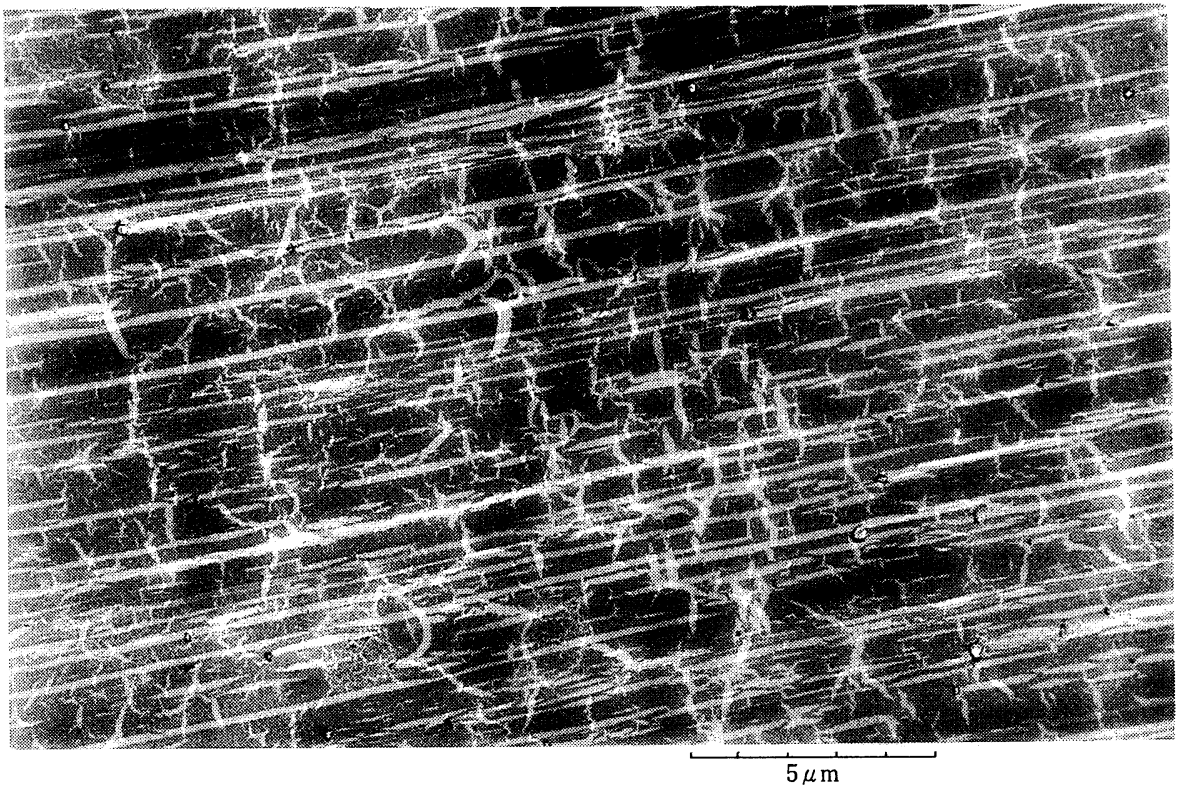

b)

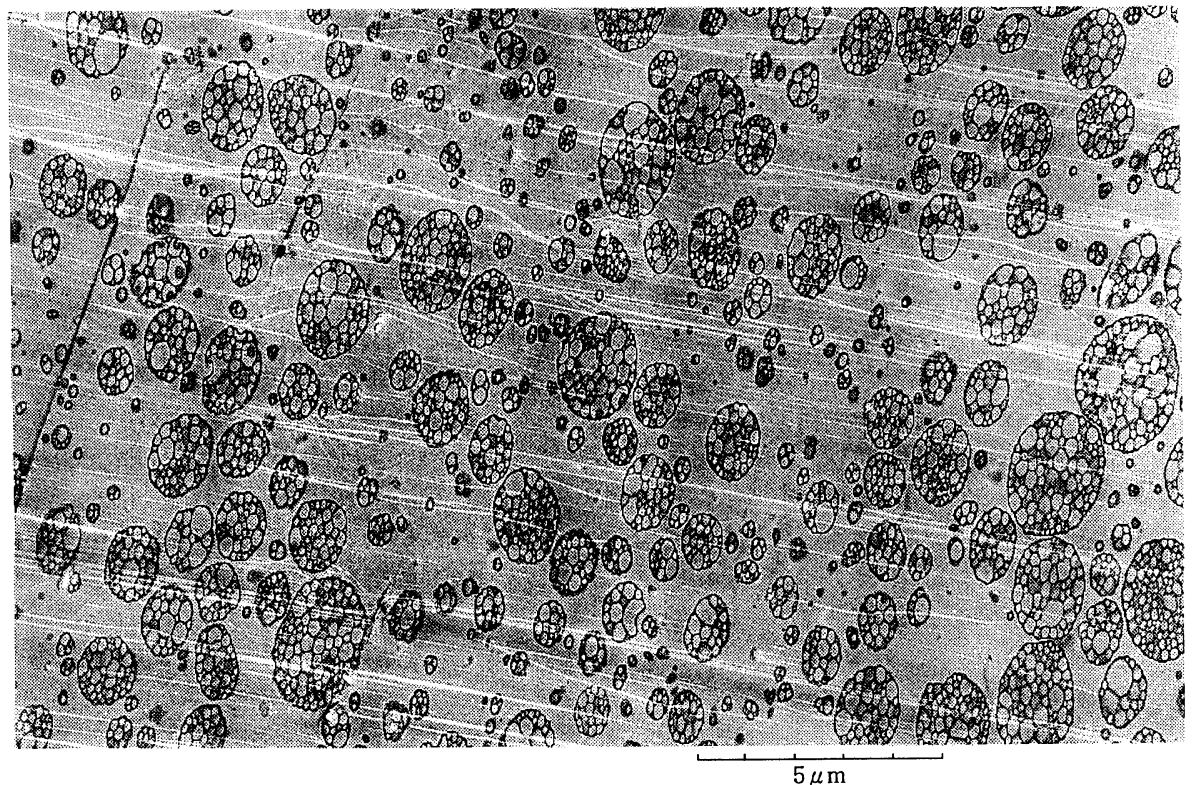

Figure 2. TEM micrographs of a) PPE/GPPS/PTFE/EBS (69/29/1/1) melt-kneaded at $330^{\circ} \mathrm{C}$ and b) HIPS/PTFE/EBS (98/1/1) melt-kneaded at $250^{\circ} \mathrm{C}$. 
erable mechanical strength at even above the melting point and undergoing small degradation at high temperatures, less than $0.1 \% \mathrm{~h}^{-1}$ weight loss at $390^{\circ} \mathrm{C} .^{7}$ This stability is indispensable to its flame retardation effect.

PTFE also exhibits very special tensile elongation behavior, $500 \%$ at room temperature to a $1700 \%$ at about $340^{\circ} \mathrm{C}$, and then falls very rapidly to about $500 \%$ at $350-360^{\circ} \mathrm{C}$ and about $100 \%$ at $400^{\circ} \mathrm{C}$ or higher, and thus shows an extremely high plasticity at temperatures just above the melting point. ${ }^{7}$

To gain in-depth knowledge about the basic characteristics of PTFE, we investigated the relationship between PTFE melting conditions and its morphology.

When PTFE was melt-kneaded with a thermoplastic resin at shearing force and temperature above the PTFE melting point, PTFE formed a highly fibrillated, oriented, and crystallized configuration in the matrix of the thermoplastic resin, thus showing a specific higher-order structure. ${ }^{8,9}$ This highly orientated crystallization may be attributed largely to the extreme plasticity of PTFE just above its melting point.

The melt-kneading of PTFE with the high-viscosity resin $\mathrm{PPE}$, at a high shear rate and at a temperature just above the PTFE melting point, resulted in a branchedfibril PTFE configuration with oriented trunk fibers and numerous branch fibers, having diameter of $0.15 \mu \mathrm{m}$ (Figure 2). ${ }^{8,9}$ This PTFE morphology is similar to the oriented crystalline "shish kebab" structure of polyethylene prepared by applying a high shearing force at high tempearature. ${ }^{10,11}$

When PTFE was melt-kneaded with low-viscosity resin HIPS at a temperature lower than the PTFE melting point a fine linear-fibril PTFE configuration was produced entirely of linear fibers of $0.05 \mu \mathrm{m}$ in diameter with no branch fibrils (Figure 2). ${ }^{8,9}$

Table I shows dimensional changes due to heat treatment $\left(150^{\circ} \mathrm{C}, 2 \mathrm{~h}\right)$ of compression molded specimens made from HIPS and from a HIPS/PTFE/EBS resin prepared by melt-kneading under the same shear at different melt-kneading temperatures. ${ }^{8,12}$ Under heat treatment, the specimen produced from the resin meltkneaded at $330^{\circ} \mathrm{C}$ (just above the PTFE melting point) underwent larger dimensional changes than that obtained melt-kneaded at $250^{\circ} \mathrm{C}$ (below the PTFE melting point). This indicates higher orientation in the former.

\section{Flame Retardancy of PTFE-Containing Resin Composi- tions}

PTFE Role in Polymer Flame Retardation. Figure 3 shows the putative steps of the combustion cycle, which starts with polymer decomposition due to heat from the source of flame, followed by evolution of volatiles from the polymer. The volatiles diffuse through the polymeric mass of the molded product, reach the product surface, diffuse into the air, and ignite, thereby sustaining the combustion cycle.

Suppressing one or more steps of this cycle should therefore lead to flame retardation. Particularly important in this regard is the suppression of polymer decomposition and internal diffusion of volatiles. ${ }^{13,14}$

In quantitative terms, combustion may be explained
Table I. Melt-kneading temperatures and dimensional changes induced by heat treatment $\left(150^{\circ} \mathrm{C}, 2 \mathrm{~h}\right)$

\begin{tabular}{lllc}
\multicolumn{1}{c}{ Resin composition } & HIPS & \multicolumn{2}{c}{$\begin{array}{c}\text { HIPS/PTFE/EBS } \\
(98 / 1 / 1)\end{array}$} \\
\hline $\begin{array}{l}\text { Melt-kneading temperature } \\
\text { Specimen }\end{array}$ & - & $250^{\circ} \mathrm{C}$ & $330^{\circ} \mathrm{C}$ \\
Thickness & 1.05 & 1.30 & 1.62 \\
Width & 0.99 & 1.18 & 0.96 \\
Length & 0.99 & 0.82 & 0.90 \\
\hline
\end{tabular}

Note: Dimensions before heat treatment, 1.00.

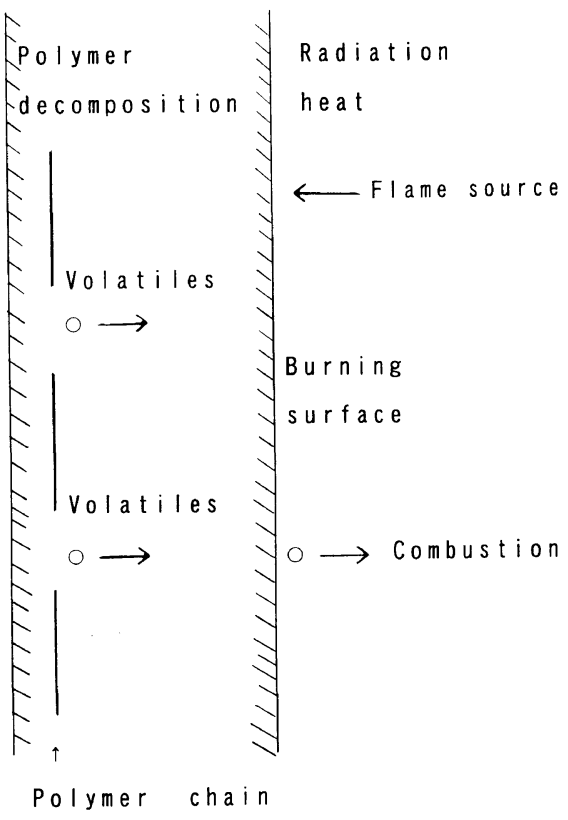

Figure 3. Putative steps of combustion cycle.

by the de Ris formula, with the horizontal flame propagation velocity $(V)$ expressed as ${ }^{15}$

$$
V=\left(2^{0.5} \lambda_{\mathrm{g}} / C_{\mathrm{c}} \rho_{\mathrm{c}} \tau\right) \times\left(T_{\mathrm{f}}-T_{\mathrm{s}}\right) /\left(T_{\mathrm{s}}-T_{\mathrm{a}}\right)
$$

where $T_{\mathrm{f}}, T_{\mathrm{s}}$, and $T_{\mathrm{a}}$ are the temperatures of flame, solid decomposition, and atmosphere, respectively; $\tau$ is the solid thickness; $\lambda, C$, and $\rho$ are heat conductivity, specific heat, and density. Subscripts $g$ and $c$ indicate gas and solid phases.

Flame retardants suppress polymer decomposition. PTFE acts as an auxiliary flame retardant. It increases polymer viscosity through entanglement with polymer molecules during combustion, to form a barrier against passage of volatiles, thereby breaking the combustion cycle.

Exposure to flame or other high heat source results in orientation release stress by oriented PTFE fibrils and therefore in a substantial increase in thickness $(\tau)$ of the solid (the molded resin composition), and thus lowering of flame propagation velocity $(V)$.

PTFE may therefore be expected to retard combustion and suppress flame dripping, through entanglement with polymer molecules and increased polymer viscosity, and thereby prevent the spread of fire to other materials.

PTFE Fibril Configurations and Flame Retardancy of PTFE-Containing Resin Compositions. ${ }^{8,9,12}$ PPE/GPPS or HIPS as a base polymer, PTFE, and a dispersing agent were melt-kneaded at $330^{\circ} \mathrm{C}$ and $250^{\circ} \mathrm{C}$, to obtain master 
Table II. Melt-kneading temperatures and flame retardation

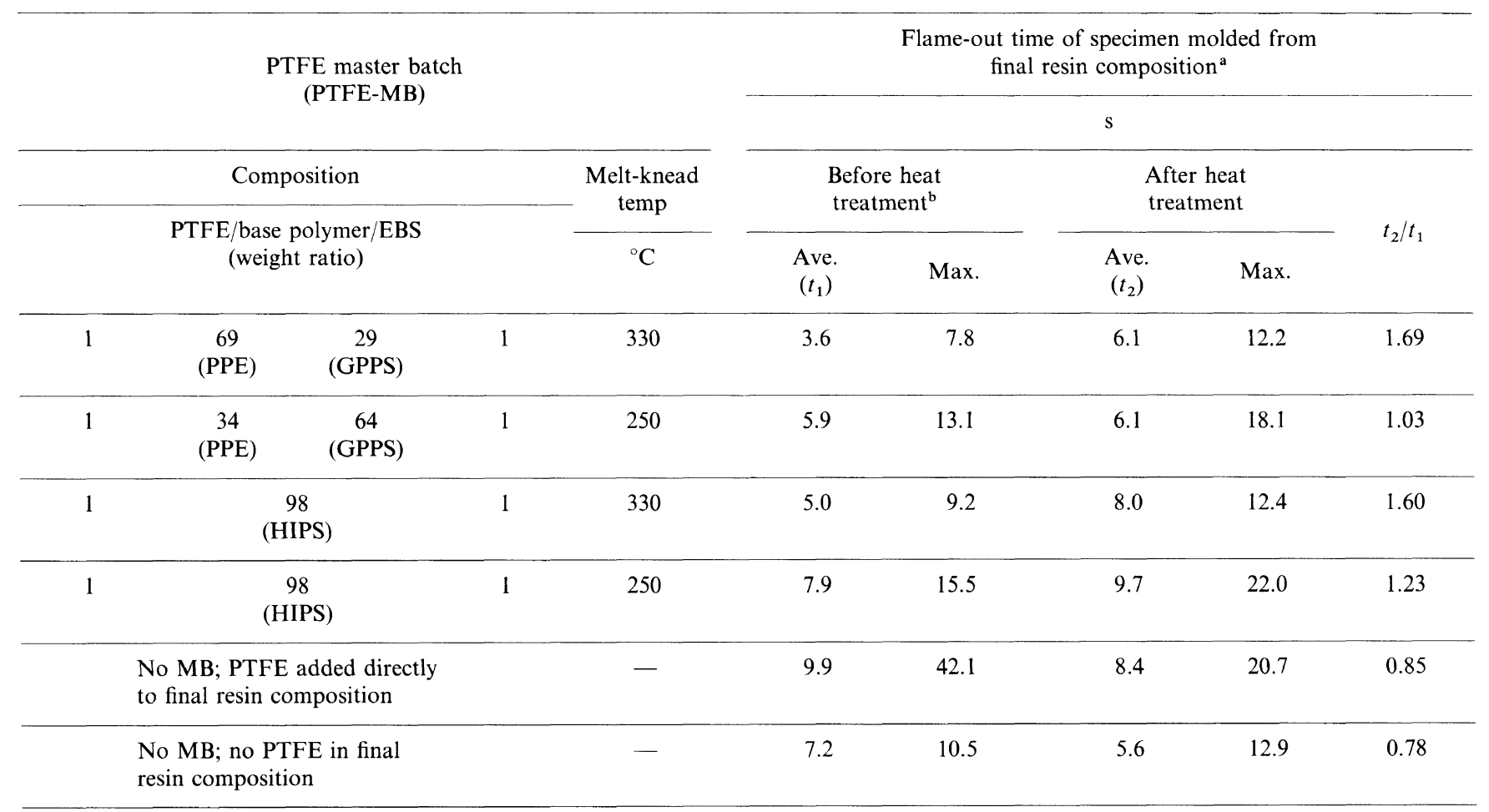

${ }^{\text {a }}$ Final resin composition: HIPS/GPPS/PPE/TPP-OH/MC/PTFE/EBS $(80 / 20 / 30 / 30 / 10 / 0.03$ or $0 / 3)$.

${ }^{\mathrm{b}}$ Heat treatment: $130^{\circ} \mathrm{C} \times 2 \mathrm{~h}$.

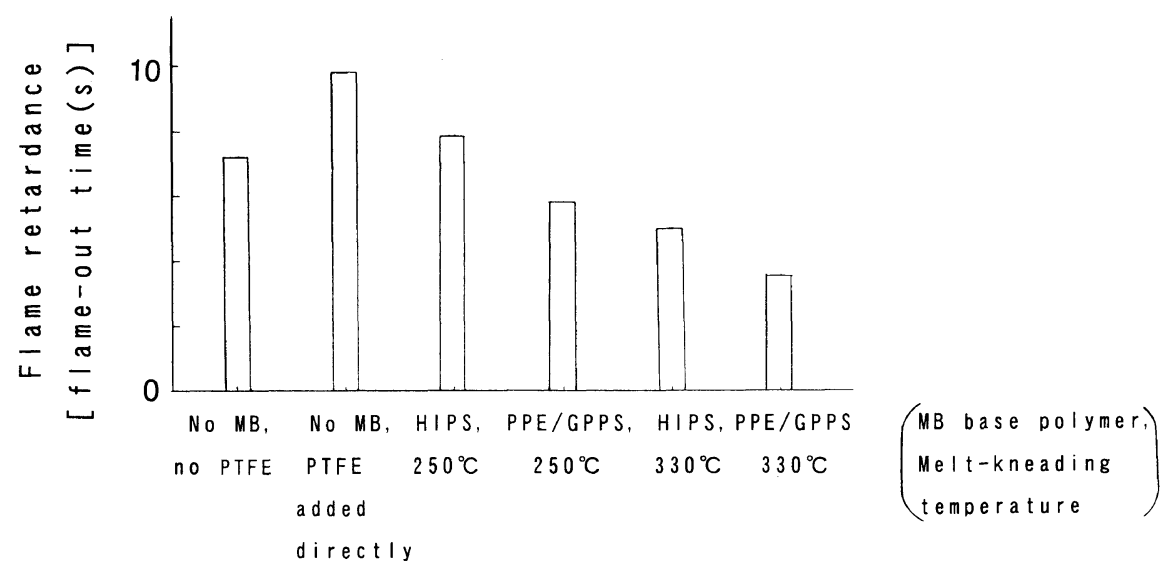

Figure 4. PTFE master batch vs. flame retardancy of final resin.

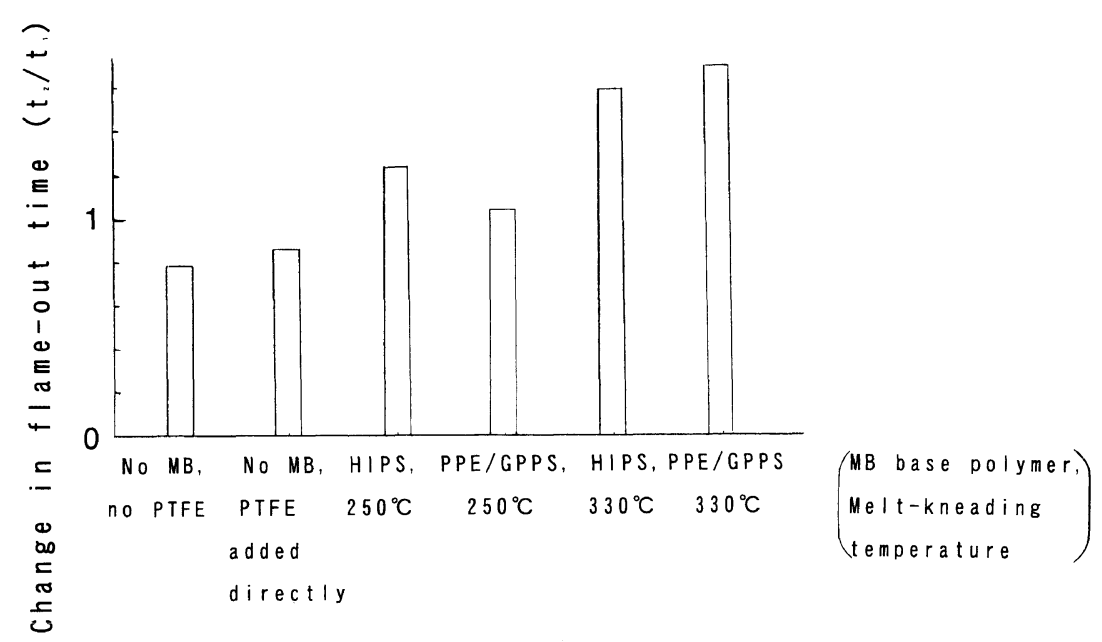

Figure 5. PTFE master batch $v s$. change in flame-out time $\left(t_{2} / t_{1}\right)$ induced by heat treatment. 
batches (PTFE-MB). The final resin was prepared by adding PTFE-MB to the preliminary resin composition.

Table II and Figure 4 summarize the relationship between PTFE melt-kneading conditions (employed in PTFE-MB preparation) and flame retardance (in terms of flame-out time) of final resin compositions, by comparing the flame-out times of final resin compositions containing (a) different types of PTFE-MB, (b) PTFE but no PTFE-MB, and (c) no PTFE.

PTFE-MB melt-kneaded at $330^{\circ} \mathrm{C}$ endowed the final resin composition with higher flame retardancy than that melt-kneaded at $250^{\circ} \mathrm{C}$. PTFE-MB containing PPE/GPPS as the base polymer imparted even higher flame retardation than that containing HIPS, which is less viscous than PPE/GPPS. These results suggest that PTFE orientation determines the flame retardancy of resin compositions containing PTFE.

Table II also shows, and Figure 5 illustrates, the flame-out time changes $\left(t_{2} / t_{1}\right)$ resulting from $130^{\circ} \mathrm{C}, 2-\mathrm{h}$ heat treatment of specimens made from the final resin. Those containing more-oriented fibrillar PTFE exhibited larger changes. This suggests heat treatment relaxes PTFE fibril orientation, thereby increasing flame-out time.

In summary, melt-kneading of a high-viscosity resin together with PTFE at a high shear rate and temperature just above the melting point of PTFE results in the formation of PTFE in a branched fibril configuration with a high orientation, which augments flame retardation of resins containing it. PTFE morphology and its high orientation were shown by transmission electron microscopy to observation, dimensional changes due to heat treatment, and flame-out time changes due to heat treatment.

\section{REFERENCES}

1. W. R. Haaf, British Pat. 1559058 (1980)

2. W. R. Haaf, U.S. Pat. 4332714 (1982).

3. W. R. Haaf, U.S. Pat. 4355126 (1982).

4. H. Nishihara, U.S. Pat. 5278212 (1994).

5. H. Nishihara, Polym. J., 29, 399 (1997).

6. H. Nishihara, W095-35346.

7. Du Pont, Wilmington, DE., The Journal of Teflon, 1, 1 (1960).

8. H. Nishihara, Polym. Prepr, Jpn., 44, 2029 (1995), English Edition: 44, E733 (1995)

9. H. Nishihara, Jpn. Pat. 6-128439 (1994) [Chem. Abstr., 121, 257152j (1994)].

10. A. J. Pennings, Kolloid-Z., 205, 160 (1965).

11. A. C. Wikjord, J. Macromol. Sci., Phys., B2, 501 (1968).

12. H. Nishihara, Jpn. Pat. 6-248189 (1994) [Chem. Abstr., 122, $189302 b(1995)]$.

13. H. Nishihara, "The Plastics - Gosei Jushi," The Japan Society of Plastics Technology, Ed., Tokyo, 1996, pp 45-54.

14. H. Nishihara, "The Technology and Application of Polymeric Materials-Market and Outlook-," CMC Co., Ltd., Tokyo, 1996, pp 432-481.

15. J. de Ris, 12th Symp. on Comb., The Comb. Inst., 1969, p 241. 